\title{
Pasteurella multocida peritonitis associated with a cat in a peritoneal dialysis patient using an automated cycler device
}

\author{
Makoto Nishina - Hidetaka Yanagi - Masahiro Koizumi · \\ Moritsugu Kimura - Takatoshi Kakuta - Masayuki Endoh • \\ Masafumi Fukagawa $\cdot$ Atsushi Takagi
}

Received: 10 January 2012/ Accepted: 8 May 2012/Published online: 6 June 2012

(C) Japanese Society of Nephrology 2012

\begin{abstract}
Pasteurella multocida ( $P$. multocida) is an aerobic Gram-negative coccobacillus, which is found as part of the natural oral flora of many animals, including most healthy cats and dogs. However, it can cause a variety of infections in humans, usually as a result of the patient being bitten or scratched by a cat or dog. There have been 22 reported cases of $P$. multocida peritonitis in patients undergoing peritoneal dialysis (PD). Of these, $66.7 \%$ occurred within 12 months of the initiation of PD. Only two cases $(11.1 \%)$ developed more than 60 months after the commencement of PD. We report a case of P. multocida peritonitis involving a 45 -year-old patient who had been undergoing continuous ambulatory peritoneal dialysis (CAPD) for 84 months without a previous history of peritonitis, who developed P. multocida peritonitis associated with a domestic cat on the very night on which he began using an automated cycler device to switch from CAPD to continuous cyclic peritoneal dialysis (CCPD). Patients maintained on PD who keep pets such as cats or dogs at home should be educated about the risk of developing peritonitis related to their domestic pets, and this warning should be repeated when such patients switch from CAPD to CCPD. Physicians should consider using empiric therapy to prevent $P$. multocida in pet-owning PD patients who present with a history of PD fluid leakage and peritonitis, especially in patients who use cycler devices.
\end{abstract}

M. Nishina $(\bowtie) \cdot$ H. Yanagi $\cdot$ M. Kimura $\cdot$ A. Takagi

Division of General Internal Medicine, Department of Internal

Medicine, Tokai University School of Medicine, 143

Shimokasuya, Isehara, Kanagawa 259-1193, Japan

e-mail: nishina@is.icc.u-tokai.ac.jp

M. Koizumi · T. Kakuta · M. Endoh · M. Fukagawa Division of Nephrology and Metabolism, Department of Internal Medicine, Tokai University School of Medicine, Isehara, Japan
Keywords Peritoneal dialysis - Pasteurella multocida . Peritonitis · Cycler device

\section{Introduction}

Peritonitis in patients undergoing peritoneal dialysis (PD) is a major cause of hospitalization, transfer to hemodialysis, and catheter removal. Staphylococcus epidermidis and $S$. aureus are the major pathogens implicated in PD peritonitis [1]. However, Pasteurella multocida (P. multocida) is a rare cause of peritonitis in PD patients.

Pasteurella multocida is a small aerobic Gram-negative coccobacillus found in the oropharynges of many animals, such as cats (50-90\%) and dogs (50-66\%) [2]. Worldwide, 22 cases of PD-associated P. multocida peritonitis have been reported to date [3-23], and in Japan, there have been only two cases $[13,23]$.

We report a patient who remained peritonitis-free during 84 months of PD who suffered from P. multocida peritonitis associated with a domestic cat on the very night on which he began to use an automated cycler device, together with a review of the literature.

\section{Case report}

A 45-year-old man who developed end-stage renal failure after IgA nephropathy had been maintained on continuous ambulatory peritoneal dialysis (CAPD) without developing peritonitis for 84 months. However, on the first night on which he used an automated cycler device to switch from CAPD to continuous cyclic peritoneal dialysis (CCPD) because of a decline of residual renal function, he became aware of dialysate leakage from the PD solution bag. 
He immediately disconnected himself from the automated peritoneal dialysis (APD) system and drained the dialysis fluid using the twin-bag system, before filling the peritoneal cavity with new solution. When he exchanged his dialysate the next morning, he noted that his effluent was cloudy. Shortly afterwards, abdominal pain developed, and he visited our hospital and was admitted.

On physical examination, he had a temperature of $38.9^{\circ} \mathrm{C}$, a pulse rate of 84 beats/min, a respiratory rate of 16 breaths/min, and a blood pressure value of $110 / 72 \mathrm{mmHg}$. His abdomen was diffusely tender and displayed rebound tenderness. His exit site and catheter tunnel showed no evidence of infection. There was no lymphadenopathy noted, which would suggest a complication of cat-scratch disease.

His laboratory data on admission showed a white blood cell (WBC) count of $12400 / \mathrm{mm}^{3}$, with $82 \%$ segmented neutrophils, and a C-reactive protein level of $1.20 \mathrm{mg} / \mathrm{dl}$. An analysis of his turbid dialysis fluid found a cell count of $3370 \mathrm{WBC} / \mathrm{mm}^{3}$, with $86 \%$ neutrophils. Gram staining of the dialysis fluid was negative. Samples of his dialysate were collected in blood culture bottles. No blood cultures were obtained. The patient was treated under a suspicion of peritonitis of unknown origin and received $1 \mathrm{~g}$ vancomycin (VCM) i.v. once and intraperitoneal ceftazidime (CAZ) $1 \mathrm{~g}$ (with a 6-h dwell time) as an empirical treatment. On the second admission day, an analysis of his peritoneal fluid demonstrated an increased WBC count of $9220 / \mathrm{mm}^{3}$, with $91 \%$ neutrophils, but his symptoms improved rapidly $48 \mathrm{~h}$ later. On the fifth admission day, an analysis of his dialysate revealed that his total WBC count had decreased to $107 / \mathrm{mm}^{3}$ (53\% neutrophils). The final culture results indicated that the organism was $P$. multocida. The isolated organism was sensitive to ampicillin, piperacillin, cefazolin, cefotiam, CAZ, levofloxacin, gentamicin, and imipenem. VCM was discontinued. The patient was switched to oral levofloxacin (200 mg/day) after 7 days of intraperitoneal CAZ treatment. There was no evidence of a decrease of peritoneal function nor ultrafiltration failure by this peritonitis episode.

The patient reported that he owned two domestic cats that were free to enter his bedroom before he started PD.

\section{Discussion}

Peritonitis remains a leading complication in PD patients. In addition, severe and prolonged peritonitis leads to peritoneal membrane failure and is a major cause of PD discontinuation and switching to hemodialysis. Therefore, preventing peritonitis is one of the most important considerations for PD patients and medical staff. Most episodes of PD-related peritonitis are caused by organisms that colonize human skin, such as $S$. epidermidis and $S$. aureus, via touch contamination or PD technique failure. In addition, a wide variety of other organisms can cause PDrelated peritonitis. However, $P$. multocida is a rare cause of PD-related peritonitis, and only 22 cases of PD-related peritonitis involving $P$. multocida have been reported to date [3-23] (Table 1).

$P$. multocida is an aerobic Gram-negative coccobacillus, which is found as part of the natural oral flora of many animals, including most healthy cats (50-90\%) and dogs (50-66 \%), and approximately $20 \%$ of cats have Pasteurella species on their claws [2, 23].

Almost all reported cases of peritonitis complications associated with this pathogen were due to puncturing of the dialysis tubing by cats or close contact with cats. Only one reported case of $P$. multocida peritonitis was due to contact with dogs [20].

It is worth noting that, although the majority of patients on PD use CAPD, most of the reported cases $(77.3 \%$ ) involved patients using a cycler device, such as CCPD or nightly intermittent peritoneal dialysis (NIPD). It is also possible that the trembling of the dialysis tube and the sound produced by the pump make the cycler device an attractive toy for cats.

In our case, the patient had remained healthy without any episodes of peritonitis during the 84 months in which he had been undergoing PD, in spite of the fact that he owned two cats. We got the impression that he took great care with his personal hygiene and dialysis procedure, and that he took appropriate measures to protect against peritonitis.

However, he developed peritonitis associated with $P$. multocida on the first day on which he used the cycler device. This episode suggests that using a cycler device is a major risk for cat-associated PD peritonitis.

In a review of $P$. multocida peritonitis, the period from the initiation of PD to the onset of peritonitis was found to be $<12$ months in most cases ( $66.7 \%$ of 18 cases), and it only occurred after more than 60 months in two cases (11.1\%). Therefore, our case was considered to be representative of this rare condition.

There was evidence of PD fluid leakage in $50 \%$ of cases. PD patients using a cycler device who also own a cat might need to be started on antibiotic therapy in order to protect against this organism, even if there is no evidence of leakage.

In our review of the literature, 20 of 22 cases recovered from peritonitis without catheter removal. $P$. multocida is susceptible to many kinds of antibiotic agent that affect Gram-negative organisms.

Empiric antibiotic combination therapy for PD-related peritonitis affects not only Gram-positive but also Gramnegative organisms. Therefore, empirical therapy involving antibiotics for Gram-negative bacteria might affect 


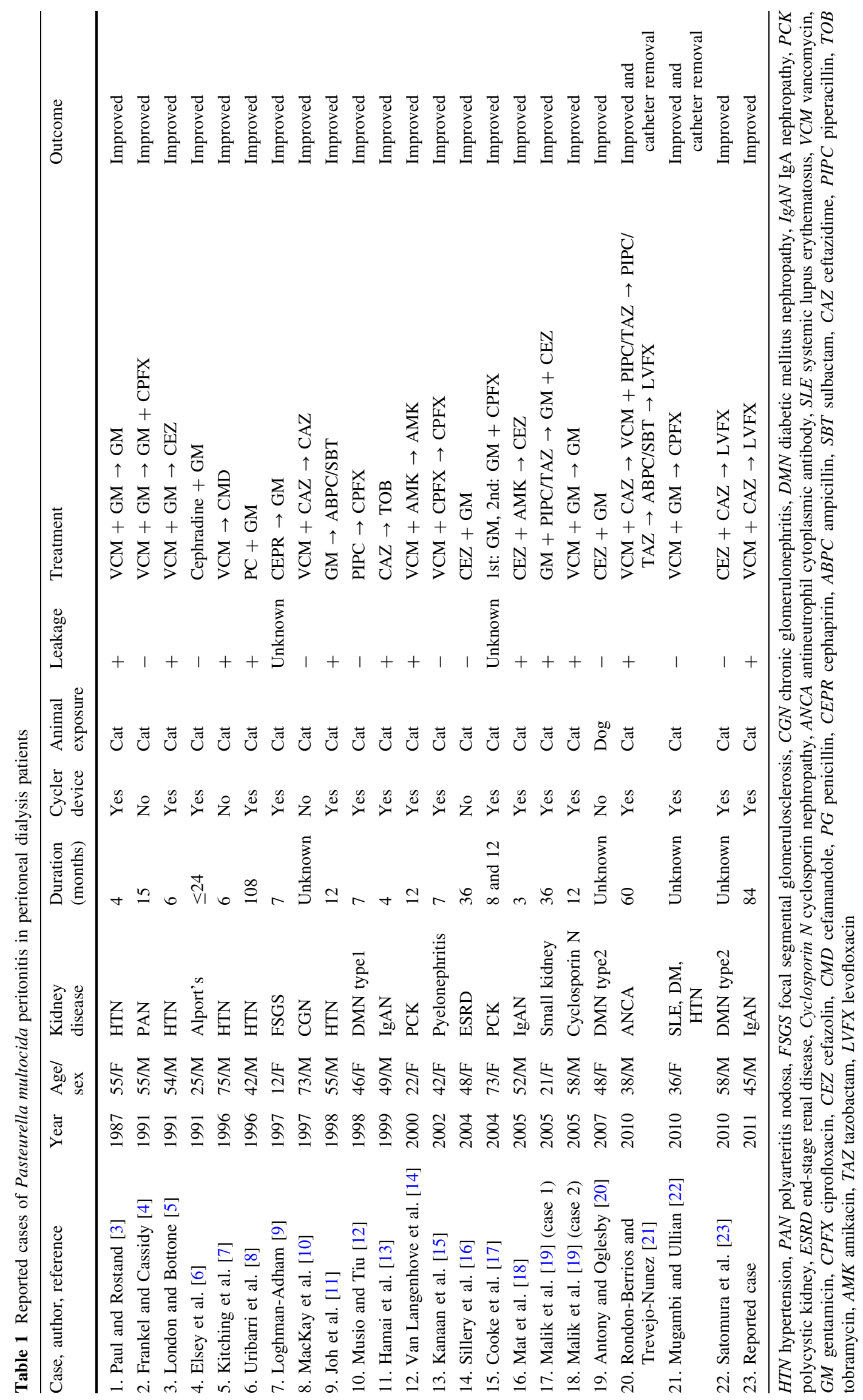


P. multocida too. In 11 of 23 cases, including our case, VCM was administered at the initiation of treatment, but was discontinued after Gram-negative bacilli were found by Gram staining or P. multocida was identified in peritoneal dialysate cultures. Gentamicin was the most frequently used antibiotic (11 cases) and was effective in each case. CAZ was used in five cases, including our case, and was effective in four cases. However, it was ineffective in one case. In this case, the treatment was changed from CAZ to piperacillin/tazobactam. Finally, ampicillin was found to be effective, and then the patient recovered. In the latest two reports $(9.1 \%)$, it was necessary to transfer the patient to an intensive care unit, as they had developed sepsis [21, 22]. The two cases were improved by changing them to appropriate antibiotic therapy and PD catheter removal.

Pasteurella species might be penicillin-resistant because they produce $\beta$-lactamase; therefore, ampicillin/sulbactam, piperacillin/tazobactam, and amoxicillin/clavulanate are recommended for the treatment of $P$. multocida [24, 25]. Patients that display cloudy effluent after being treated with appropriate antibiotics for 5 days have refractory peritonitis and should have their catheter removed [26].

In conclusion, $P$. multocida peritonitis is rare in PD patients, and only 22 such cases have been reported. Its transmission almost always involves a cat biting or scratching the PD tubing or bags.

Patients maintained on PD who keep pets such as cats or dogs at home should be educated about the possibility of developing peritonitis related to their domestic pets. When these patients switch from CAPD to CCPD, they should be given information about the risk of pet-associated peritonitis.

Physicians should consider empiric therapy against P. multocida in pet-owning PD patients who present with a history of PD fluid leakage and peritonitis, especially in patients who use cycler devices.

\section{References}

1. Vargemezis V, Thodis E. Prevention and management of peritonitis and exit-site infection in patients on continuous ambulatory peritoneal dialysis. Nephrol Dial Transplant. 2001;16:106-8.

2. Weber DJ, Wolfson JS, Swartz MN, Hooper DC. Pasteurella multocida infections. Report of 34 cases and review of the literature. Medicine (Baltimore). 1984;63:133-54.

3. Paul RV, Rostand SG. Cat-bite peritonitis: Pasteurella multocida peritonitis following feline contamination of peritoneal dialysis tubing. Am J Kidney Dis. 1987;10:318-9.

4. Frankel AH, Cassidy MJD. Pasteurella multocida peritonitis in CAPD: beware of the cats. Perit Dial Int. 1991;11:184-5.

5. London RD, Bottone EJ. Pasteurella multocida: zoonotic cause of peritonitis in a patient undergoing peritoneal dialysis. Am J Med. 1991;91:202-4.
6. Elsey RM, Carson RW, DuBose TD Jr. Pasteurella multocida peritonitis in an HIV-positive patient on continuous cycling peritoneal dialysis. Am J Nephrol. 1991;11:61-3.

7. Kitching AR, Macdonald A, Hatfield PJ. Pasteurella multocida infection in continuous ambulatory peritoneal dialysis. $\mathrm{N} Z \mathrm{Z} \mathrm{Med}$ J. 1996;109:59.

8. Uribarri J, Bottone EJ, London RD. Pasteurella multocida peritonitis: are peritoneal dialysis patients on cyclers at increased risk? Perit Dial Int. 1996;16:648-9.

9. Loghman-Adham M. Pasteurella multocida peritonitis in patients undergoing peritoneal dialysis. Pediatr Nephrol. 1997;11:353-4.

10. MacKay K, Brown L, Hudson F. Pasteurella multocida peritonitis in peritoneal dialysis patients: beware of the cat. Perit Dial Int. 1997;17:608-10.

11. Joh J, Padmanabhan R, Bastani B. Pasteurella multocida peritonitis following cat bite of peritoneal dialysis tubing. With a brief review of the literature. Am J Nephrol. 1998;18:258-9.

12. Musio F, Tiu A. Pasteurella multocida peritonitis in peritoneal dialysis. Clin Nephrol. 1998;49:258-61.

13. Hamai K, Imai H, Ohtani H, Komatsuda A, Kodama T, Wakui H, et al. Repeated cat-associated peritonitis in a patient on automated nocturnal intermittent peritoneal dialysis. Clin Exp Nephrol. 1999;1:59-61.

14. Van Langenhove G, Daelemans R, Zachée P, Lins RL. Pasteurella multocida as a rare cause of peritonitis in peritoneal dialysis. Nephron. 2000;85:283-4.

15. Kanaan N, Gavage P, Janssens M, Avesani V, Gigi J, Goffin E. Pasteurella multocida in peritoneal dialysis: a rare cause of peritonitis associated with exposure to domestic cats. Acta Clin Belg. 2002;57:254-6.

16. Sillery J, Hargreaves J, Marin P, Lerma E, Kuznia C, Abbe C. Pasteurella multocida peritonitis: another risk of animal-assisted therapy. Infect Control Hosp Epidemiol. 2004;25:5-6.

17. Cooke FJ, Kodjo A, Clutterbuck EJ, Bamford KB. A case of Pasteurella multocida peritoneal dialysis-associated peritonitis and review of the literature. Int J Infect Dis. 2004;8:171-4.

18. Mat O, Moenens F, Beauwens R, Rossi C, Muniz-Martinez MC, Mestrez F, et al. Indolent Pasteurella multocida peritonitis in a CCPD patient. 25 years of "cat-bite peritonitis": a review. Perit Dial Int. 2005;25:88-90.

19. Malik A, Al Aly Z, Mailey KS, Bastani B. Pasteurella multocida peritoneal dialysis-associated peritonitis: a report of two cases and review of the literature. J Nephrol. 2005;18:791-3.

20. Antony SJ, Oglesby KA. Peritonitis associated with Pasteurella multocida in peritoneal dialysis patients - case report and review of the literature. Clin Nephrol. 2007;68:52-6.

21. Rondon-Berrios H, Trevejo-Nunez GJ. Pets or pest: peritoneal dialysis-related peritonitis due to Pasteurella multocida. J Microbiol Immunol Infect. 2010;43:155-8.

22. Mugambi SM, Ullian ME. Bacteremia, sepsis, and peritonitis with Pasteurella multocida in a peritoneal dialysis patient. Perit Dial Int. 2010;30:381-3.

23. Satomura A, Yanai M, Fujita T, Arashima Y, Kumasaka K, Nakane C, et al. Peritonitis associated with Pasteurella multocida: molecular evidence of zoonotic etiology. Ther Apher Dial. 2010;14:373-6.

24. Lion C, Lozniewski A, Rosner V, Weber M. Lung abscess due to $\beta$-lactamase-producing Pasteurella multocida. Clin Infect Dis. 1999;29:1345-6.

25. Jorgensen JH, Hindler JF. New consensus guidelines from the Clinical and Laboratory Standards Institute for antimicrobial susceptibility testing of infrequently isolated or fastidious bacteria. Clin Infect Dis. 2007;44:280-6.

26. Li PK, Szeto CC, Piraino B, Bernardini J, Figueiredo AE, Gupta A, et al. Peritoneal dialysis-related infections recommendations: 2010 update. Perit Dial Int. 2010;30:393-423. 